\title{
Malaria elimination: needs assessment and priorities for the Future
}

\author{
Amal A. El-Moamly \\ Parasitology Department, Faculty of Medicine, Suez Canal University, Egypt
}

\begin{abstract}
When the World Health Organization announced the goal of global eradication of malaria in 2007, questions were raised about the naivety of this proposition. Since then, experts have been divided about this goal. Some scientists suggest that when defeating malaria, elimination is a worthy and challenging aim, but this has to be done with modesty and thorough analysis. Others believe that it is time to repeat the experience of smallpox eradication and to open a whole new era for public health, the eradication of many diseases. The opposing view suggests that raising expectations and failing again may set malaria control back, rather than advance its cause. This literature review focuses on malaria elimination. It summarizes the history of malaria elimination, its success factors and reasons for failure, and the controversial issues in malaria elimination. The collected articles on the challenges of elimination, and the technical and financial feasibility that countries must appreciate before proceeding, are identified. Also, this review discusses the current global strategy to eliminate malaria and highlights the main concerns for future plans aimed at elimination. These plans foresee improving currently available diagnostic methods, therapeutic and prophylactic agents and protocols, vector control procedures, vaccine development progress, and other operational tools and approaches. Finally, this review addresses a number of research priorities in the present stage of the fight against malaria.
\end{abstract}

Key words: Malaria; elimination; eradication; control; diagnostics; vaccine; treatment; Plasmodium

J Infect Dev Ctries 2013; 7(11):769-780. doi:10.3855/jidc.3079

(Received 15 October 2012 - Accepted 28 May 2013)

Copyright (C) 2013 El-Moamly. This is an open-access article distributed under the Creative Commons Attribution License, which permits unrestricted use, distribution, and reproduction in any medium, provided the original work is properly cited.

\section{Introduction}

When the World Health Organization (WHO) announced the goal of global eradication of malaria in 2007, and the hope that eradication could occur within a few decades, questions were raised about the naivety of this proposition. Since then, experts have been divided about this goal. Should the international community recognize, support, and work for the vision of a malaria-free world? Some have proposed that in the fight against malaria, elimination is a worthy, challenging and achievable aim, but cautioned that efforts must be conducted in a modest and balanced way and must be accompanied by rigorous analysis. Other experts believe that it is time to repeat the experience of smallpox eradication [1]; still others with more impressive dreams have argued that a whole new era for public health has begun with the eradication of many microbial diseases. Malaria was a top priority among these diseases, so the usefulness and feasibility of its eradication was of great interest to researchers. The counterview proposed that raising expectations and failing may set malaria control back, rather than advance its cause [2]. The risks may outweigh the benefits of eradication, leading to a resurgence of malaria if funding support changes or political commitment wanes. The emergence of new pathogens might also constitute a biosafety risk that could result from the global eradication of malaria. Scientists continue to discuss which goal is more valuable in defeating malaria: control or elimination. They argue that elimination efforts must not detract from existing effective control measures in endemic regions. It has also been suggested that one practical way forward might be to shrink the malaria map [2]. What is certain, in the view of many experts, is that pursuing malaria elimination will change priorities for treatment, chemotherapeutic prophylaxis, vector control, and malaria vaccination. Some think malaria will only be a truly eliminable disease when an effective vaccine is fully developed [1]. Generally, experts believe that the strategy of shrinking the malaria map, combined with a crucial insistence on control in countries with the largest burdens of malaria and intensive research to improve currently available diagnostics, drugs, insecticides, and vaccines, can produce concrete progress towards elimination.

The ultimate aim of this review was to improve the outcomes of human efforts in the fight against malaria. The main question this review aimed to answer was whether malaria elimination is an achievable and 
worthwhile objective. The objectives of this review were thus to review the literature and provide an update on current and previous global efforts to eliminate malaria, to discuss the controversies surrounding these efforts, and to suggest priorities for future elimination strategies aimed at achieving the dream of a malaria-free world.

\section{Definition of elimination: Difference between control and elimination}

The terms control, elimination, and eradication have been reviewed; it was found that they are often used changeably, and this results in confusion [3]. Eradication was formerly used to describe what is currently called elimination, but is currently used to denote the "permanent reduction to zero of the worldwide incidence of infection caused by a specific agent as a result of deliberate efforts," with further interventions no longer needed [3]. According to WHO, malaria elimination was defined as "interrupting local mosquito-borne malaria transmission in a defined geographical area to zero incidence of locally contracted cases, although imported cases will continue to occur and continued intervention measures are required" [4]. Some believe WHO's literal definition of elimination is not applicable anywhere because small outbreaks from imported cases sometimes occur in countries that have achieved elimination [5]. In 2006, WHO highlighted this point and proposed an operational definition of elimination as "nationwide per year fewer than three 'epidemiologically linked' cases of a malaria infection without an identifiable risk factor other than local mosquito transmission, for three consecutive years" [4]. Recently, the definitions of the terms control, elimination, and eradication were reviewed. A new meaning for elimination was proposed as "a state where interventions have interrupted endemic transmission and limited onward transmission from imported infections below a threshold at which risk of re-establishment is minimized. Both capacity and commitment to sustain this state indefinitely are required" [6]. This new definition acknowledges that maintaining zero incidence of locally contracted cases is not always an achievable aim.

On the other hand, WHO defined malaria control as reducing the disease burden to a level at which it is no longer a public health problem [4]. In 2010, Cohen et al. [6] introduced a new expression, controlled lowendemic malaria, defined as "a state where interventions have reduced endemic malaria transmission to such low levels that it does not constitute a major public health burden, but at which transmission would continue to occur even in the absence of importation." In this respect, WHO categorizes countries in four program phases on the basis of slide positivity rates and incidence rates: control, pre-elimination, elimination, and prevention of re-introduction. A country is considered to be in the elimination phase when it has a parasite incidence per year of less than one per each one thousand people at risk [7]. Accordingly, 109 countries in the world are malaria-free (Egypt is a recent example), 67 are in the malaria-controlling phase, and 32 are in the malariaeliminating phase [8-9]. In their 2010 review article, Feachem et al. highlighted various definitions used in malaria elimination and control [9].

\section{History of malaria elimination}

Efforts to control malaria widely started in the final years of the nineteenth century, when the Plasmodium parasite was discovered and malaria transmission by mosquito vectors was reported [10]. During the first half of the twentieth century, while malaria was endemic in large parts of the world, including developed countries such as the United States of America and Western European countries, little progress was made toward elimination, partly because of World Wars I and II. However, when the Global Malaria Eradication Program (GMEP) was launched in 1955, all countries outside of Africa where malaria was endemic were working toward or about to begin working toward malaria elimination [9]. When financial support for the GMEP collapsed and the program stopped in 1969 , most countries that had successfully achieved elimination continued to be malaria free [9]. However, many of the countries that remained endemic were left with fewer well-supported programs, chloroquine and DDT resistance, and insufficient funding and guidance commitments [11]. This situation led to significant increases in worldwide malaria in the 1970s and 1980s [11]. Other reasons for the re-emergence of malaria were the change in political situations in certain countries, as seen after the collapse of the Soviet Union with the consequent damage to the economy and health system, and reappearance of malaria endemicity [12]. With the recently renewed interest in eradication, the scale-up of malaria control driven by the rise in funding and new strategies to combat malaria [2] have lead to progress toward elimination in many countries since the early years of the twenty-first century [13-14]. Currently, although malaria remains endemic in 99 countries, 109 are free of malaria [10] and $50 \%$ of the 
world's population now lives in malaria-free areas compared to $30 \%$ in 1950 [ 8].

A new strategy for malaria eradication (the threepart strategy) was recently developed with a consensus to ultimately bring about malaria elimination $[8,13-$ 14], and is now widely endorsed [15-17]. This strategy includes (1) aggressive use of malaria control measures in to reduce the transmission and mortality rates in highly-endemic countries with the highest morbidity and mortality rates; (2) progressive gradual elimination of malaria from the margins of endemic regions inwards (shrinking the malaria map); and (3) research to discover and develop novel interventions, delivery tools and technologies (i.e. research into vaccines and novel drugs, diagnostic tools, vector control methods, and other interventions and delivery procedures) accessible to all at-risk populations [14]. Considerable efforts and investments have been devoted to part one of this strategy, which focuses on tough control measures in endemic countries; this has produced a substantial increase in effective methods and procedures [18] and a considerable decrease in mortality and morbidity rates in some endemic regions [14]. Also, part three of strategy, which emphasizes research into novel interventions and tools, has benefited from scaling-up in investments and activities in the past decade, and an agenda for research in malaria eradication has been established [15]. In contrast, part two of the new strategy, which addresses shrinking the malaria map, has received less consideration, and as a result the evidence-based data available to guide decision makers remains inadequate [19]. However, some feel that reallocating investment priorities from part one of the strategy to part three (i.e., from aggressive control in highly endemic countries to shrinking the malaria map), is not a reasonable move and cannot be justified [9].

\section{Malaria elimination: needs assessment for the future and priorities for the next stage}

Many believe that in planning the elimination agenda for the next phase, the first point to emphasize is that efforts to shrink the malaria map should in no way detract from control in highly endemic countries or from the development of new interventions and delivery methods [9]. In fact, the activities that a malaria program should undertake to maintain controlled low-endemic malaria should be well established before an elimination effort is launched [10]. However, greater attention to shrinking the malaria map is also crucial for global malaria eradication. Ensuring global agreement on elimination is mainly the role of WHO, but with the support of additional partners and stakeholders such as the Global Fund, the United Nations Children's Fund (UNICEF), and others [9]. A strong commitment to all three elements of the three part-strategy is a fundamental aspect of the elimination of malaria. However, some efforts to improve the implementation of the strategy require attention to cope with recent changes in the epidemiology of malaria in settings where elimination is the goal. Therefore, the requirements to achieve the required outcomes of this strategy should be prioritized in the near future. Based on the controversial risks, benefits, and challenges of elimination, the objectives presented in the following sections of this review constitute the top priorities in the current stage of the battle for malaria elimination.

\section{Producing feasibility assessments}

According to Marsh [45] although thorough assessments of financial feasibility of the elimination process are critical, Oliver Sabot and colleagues [20] have indicated the huge evidence gap especially in estimating the marginal costs and the financial benefits on the long run and the threshold benefits which are difficult to assess. Sustained efforts to develop a feasibility assessment in a user-friendly form would be of great support to malaria program workers in countries that are pursuing elimination, and such assessments could guide policy makers in these countries in ways to move from control to elimination [9]. Full documentation of the experiences in different countries with past and present elimination practices can help malaria-eliminating countries learn from other trials [16]. The economic aspects of elimination should be studied carefully; the first priority is to increase our understanding of the costs of implementing elimination and sustaining it by avoiding reintroduction, and to compare these costs with those of maintaining controlled low-endemic malaria [36]. A comprehensive assessment of the benefits of elimination locally, regionally, and worldwide would also be very useful [9]. Moreover, better evidence guidelines about the best use of interventions in specific elimination situations are also important [21].

\section{Improving passive case detection}

Data are routinely received in passive case detection systems based on specific rules. These systems need a health-care provider or health facility to report certain diseases or disorders on a regular basis and at specific times (weekly, monthly, or yearly) $[22,36]$. Because of limitations in the accuracy 
of the diagnostic methods and efficacy of treatment, passive case detection cannot detect all new infections in the population, and can only identify, at best, $40 \%$ of all new cases [23]. To support these activities, additional efforts are needed to optimize passive case detection. This may not be achieved by feasible and affordable access to malaria diagnosis and treatment alone, but may also require improvements in health care capacity, laboratory tools and health-seeking behavior of patients, and by enhancements in the reporting processes at private and public facilities [36].

\section{Reducing the importation of malaria by active case detection}

The importation of malaria is inevitable, even in countries that have eliminated malaria long ago. If improved health care systems exist and broad diseasereporting mechanisms are ensured, passive case detection can be sufficient to prevent resurgence from imported cases or can detect new cases promptly if domestic transmission is low [36]. However, in most malaria-endemic countries contemplating elimination, the high vectorial capacity might result in significant transmission even if the rate of importation is low [24]. Therefore, to eliminate malaria, the flow of imported infections must be slowed through border screening, regional and cross-border initiatives, proactive case detection, and treatment in high-risk migrants and travelers, before it leads to a resurgence of the disease [13].

\section{Killing the parasite with appropriate treatment Primaquine: advantages and disadvantages}

Detecting both symptomatic and asymptomatic patients and killing all forms of the parasite they carry are required to eradicate the parasite in a human reservoir host [36]. Primaquine is recommended by WHO for elimination [25], and 8-aminoquinoline tafenoquine [26] is also very successful against the mature gametocytes of Plasmodium falciparum. However, the effects of both on $P$. vivax and $P$. ovale liver hypnozoites are variable [27]. Moreover, they may cause hemolysis in glucose-6-phosphate dehydrogenase (G6PD)-deficient patients [28], even at low doses and with mild G6PD deficiency [6,29]. Feachem et al. (2010) indicated thata the unavailability of a quick, point-of-care G6PD test for persons at risk of hemolysis is a challenge [9]; diagnostic trials of such tests have recently started.

Drugs that act against developing $P$. falciparum gametocytes such as artemisinin combinations can reduce the chances of transmission [30]. Adding primaquine to the combination in order to enhance the blockage of transmission is not recommended, especially in patients with acute disease; however, patients with chronic infections or recrudescence usually have gametocytes in their blood [30] and can thus benefit from the addition of primaquine to artemisinin combination therapy or treatment with other drugs to eradicate gametocytes [30]. More evidence is needed to confirm the advantages of adding a single dose of primaquine in light of the possibly harmful hemolytic effect in these patients [31,36].

In $P$. vivax, $P$. ovale or $P$. malariae infections, there is no need to target gametocytes in order to eradicate the blood stages, which show susceptibility to the recommended drugs [32]. Primaquine is considered the only anti-hypnozoite drug, and can thus prevent relapse and emerging onward transmission [33]. The standard dose of primaquine for two weeks has not always been proven useful for eliminating a relapse crisis $[25,33]$. Other radical drugs used at high doses for prolonged periods may have greater a effect, but the evidence of their effectiveness has not yet been fully convincing; moreover, side effects and compliance with these therapeutic regimens are controversial issues [33-34]. Radical drugs with fewer safety concerns, a longer half-life and better compliance by patients are urgently needed because the lack of treatment options remains the main impediment to the elimination of $P$. vivax $[35,36]$.

\section{Mass treatment}

Treating patients who are detected by passive case detection only is not likely to eradicate the parasite in a human reservoir or stop forward transmission in most situations, even with radical treatment (assuming that compliance with treatment is perfect) [36]. However, compliance with primaquine treatment is difficult because the drug needs to be taken for 14 days or longer. From an operational perspective, the difference between maintaining low endemic malaria under control and eliminating the disease lies in the system of detecting, reporting and responding to any imported cases and potential outbreaks. Because additional active surveillance programs to detect asymptomatic carriers are expensive, other options such as mass drug administration (MDA) have been proposed to reduce the parasite in human reservoir hosts [25]. The MDA treatment regimens in use differ widely, but primaquine is almost always included as a radical drug. WHO does not strongly support MDA [25], but has proposed that it should be used in 
specific geographic settings or for well-defined target population groups. However, MDA may be useful to prevent or restrict outbreaks and avoid imported malaria transmissions, particularly in $P$. falciparum infections. To reduce the risk of developing resistance, drug combinations that are different from first-choice agents are recommended. The combination should include an agent with a long half-life, preferably able to destroy sexual forms [25]. In $P$. vivax infection, MDA may not completely eradicate liver hypnozoites. Therefore, additional strategies should be examined to deal with relapses and focal infections $[35,36]$.

\section{Implementing elimination-specific vector control activities}

Rigorous entomological surveillance

Another concern globally is the rapid spread of insecticide resistance. Failure to successfully eliminate the disease or the collapse of control program may result in weak intervention measures and more resistant mosquito populations. Therefore, vector control strategies designed for specific settings during an elimination program should differ from those used in control programs [36]. The identification of highrisk regions and use of geostatistical analysis of incidental cases [25], along with thorough entomological surveillance, are essential to the continuous assessment of the likelihood of transmission and vector competence, and to efforts to monitor insecticide resistance and replace the dominant vector species [36,37]. In Morocco, for example, entomological studies found that larval control measures decreased the vectorial capacity of anopheline mosquitoes, resulting in a low resurgence rate despite the high number of individuals who carry gametocytes [38]. Insecticide resistance in vector larvae and adults creates a major obstacle to elimination, and overcoming these obstacles requires extensive, continuous biosurveillance [8,38]. In South Africa, for example, increased vector resistance to pyrethroids, which became widely ineffective, prevented this country from reaching the preelimination stage after DDT was reintroduced [38]. Resistance to common insecticides in central Sudan, a low endemic region, has made it impossible to block successful transmission [38].

Unfortunately, in most countries, entomologic indices such as vector sensitivity to insecticides and entomologic inoculation rates (EIRs) and the bionomics of anopheline mosquitoes are not routinely used. Entomologic assessments, particularly EIRs which vary considerably depending on temperature, rainfall, humidity and other environmental parameters - are frequently done by research institutions only and are often not well coordinated with prevention action plans in endemic countries. The continuous assessment of these measures is crucial to evaluate progress in malaria elimination, and should be used in control programs designed to decrease transmission in endemic countries. Moreover, entomological, parasitological and human surveillance must be performed at the same time in the same localities, and the shared information must be used to improve control strategies. Entomologic studies require skilled personnel to collect and identify anopheline species and subspecies in the habitats of the larvae and adults. Using sound sampling techniques before, during and after the elimination program is important to ensure that the information collected is accurate. Accordingly, the African Network on Vector Resistance provides useful information on the standardized testing of mosquito sensitivity to insecticides.

\section{Not only indoor but also outdoor biter control}

Control programs generally focus interventions aimed at indoor biting mosquitoes, which are the most efficient vectors. However, all vectors, including those that bite, outdoors should be targeted in order to achieve complete cessation of endemic transmission. When the GMEP was started by WHO, outdoor biting and resting vectors were considered of minor importance because they were thought to be limited to a few Anopheles species [39]. Later on in the program, vector behavior, such as the emerging resistance to DDT, was recognized as affecting transmission [40]. The contribution of these outdoor biting vectors to the overall receptivity of transmission foci remains unclear. Although bed nets saturated with insecticides have been shown to affect transmission by outdoor biting vectors [40], further research is needed to establish the most effective methods for entomological surveillance and to identify additional vector control measures that might be necessary to completely abort transmission. The GMEP has suggested that after the attack phase, most vector control measures can be scaled down [39]. Therefore, most malaria control programs considering elimination will need to strengthen their entomological activities and expertise considerably, not only to identify receptive foci but also to be able to make evidence-based decisions about what the best strategies to control vectors are and when to scale them down safely [36]. Regular adult and larval surveillance are essential to the effectiveness of elimination programs. Studying adult 
habits (e.g., emergence, swarming, mating, biting behavior, flight range, longevity, and hibernation) in addition to those of the larval phase (e.g., breeding places, animal fauna, plants, water and food in the environment) is important to prepare rapid responses in dynamic ecological settings. Research evidence about the effectiveness and feasibility of nontraditional uses of insecticides is needed. The use of washresistant insecticide-treated clothing should be evaluated for individuals exposed to infection outdoors due to their occupation and for at-risk groups such as in refugee camp inhabitants. In addition, the ability of repellents such as DEET, picaridin, citronella, botanicals and others to protect individuals exposed to outdoor vectors outside the homes should be evaluated.

\section{Improving diagnostic laboratory techniques}

New diagnostic methods and approaches are increasingly important in efforts to improve surveillance, the precision of transmission data, and the detection of cases of malaria infection [36]. The ability to reliably diagnose malaria infection is fundamental to both the management of individual patients as well as public health efforts to control the disease and prevent further spread in the community via local vectors. The working group on malaria and the UN Millennium Project took a number of measures into account in their proposed global plan, which involves the measurable target to reduce malaria morbidity and mortality by $75 \%$ by 2015 from the 2005 baseline level. One of the priorities is support for an intensive research program to develop new, improved, and affordable tools for diagnosis. WHO has started conversations with scientists, clinicians, and producers of diagnostic tests for malaria about the possibilities of developing rapid, accurate, valid, reliable, and cost-effective tests. Among the stipulations for these rapid tests are the capability to detect 100 parasites $/ \mu \mathrm{L}$ in all Plasmodium species, and the ability to perform semi-quantitative measurements to monitor drug resistance. Test sensitivity and specificity are not the only priorities; other diagnostic challenges such as diagnosis in passive carriers or asymptomatic patients, diagnosis in the field, diagnosis of mixed infections and imported malaria, and the different diagnostic requirements in developed non-endemic and less-developed endemic countries are also important.
Microscopy and rapid diagnostic tests

The sensitivity of well-prepared blood films for light microscopic examination and high-accuracy rapid diagnostic tests are adequate to detect malaria parasites and confirm the diagnosis in symptomatic patients, which is required for the management of malaria in endemic regions [25]. However, asymptomatic carriers and patients with mild clinical manifestations and low parasitic density in lessendemic countries are more common, and detecting infection in these people is more important because these asymptomatic carriers will continue to cause onward transmission silently. The ability of both types of test to detect low-parasite-density infections is limited in these situations [41]. The reliability of microscopy is a problem in the diagnosis of imported malaria in non-endemic countries, where it is a challenge for most laboratories to maintain their proficiency in reading malaria smears. The accepted patterns of morphological appearance of malaria species have changed, probably due to drug pressure, strain variation and methods of blood collection as well as mixed infections, and these factors have created further challenges to microscopic diagnosis that cannot be solved by simply consulting an atlas. Moreover, quality assurance protocols for microscopy are difficult to implement in elimination areas. Although microscopic diagnosis is sensitive and specific and remains the standard method for diagnosing malaria, it is not universally available, not considered a rapid diagnostic method, and requires a high level of expertise. Fluorescence microscopy was shown to improve the sensitivity, but not the specificity, of habitual microscopy-based procedures. Because of concerns about the sensitivity of rapid diagnostic tests in infections with low parasite densities, and their uncertain specificity for species other than $P$. falciparum, standardized quality assurance protocols are needed to confirm the diagnosis in the large numbers of suspicious negative results [36].

\section{DNA polymerase chain reaction techniques}

Polymerase chain reaction (PCR)-based methods for Plasmodium DNA are very sensitive and useful for determining the culprit species in malarial infection. They provide a more sensitive means of testing than microscopy or rapid diagnostic tests, but better standardized and field-applicable methods with robust quality assurance mechanisms are needed [41]. Retesting specimens that were negative on microscopic examination and rapid diagnostic testing 
with pooled DNA-PCR techniques [42] or loop attenuated isothermal amplification have yielded promising results, but more research is needed before strong recommendations can be provided [36]. PCR methods are especially valuable for large-number specimens because dried blood spots can be tested, and even a single sample of low-grade parasitemia can be detected in a 50-sample pool, which saves resources and provides a cost-effective procedure in many settings. Quantitative PCR (real-time PCR) is also valuable in determining the intensity of infection and monitoring therapeutic success. This method offers a good alternative to other standard techniques, which are less helpful in follow-up and do not provide information on therapeutic efficacy. However, PCR techniques are expensive, not readily available at most hospital and clinical laboratories, and require a long time to produce results $[42,43]$. They are not used in most places where malaria is endemic, and are not amenable to field and point-of-care diagnosis; moreover, they require highly trained staff. Using DNA-PCR as a point-of-care method in field settings would provide high diagnostic accuracy but unfortunately, it seems that this technique will not be available in the near future. At present, DNA-PCR appears more suitable for the detection of active malaria cases in limited specialized centers [42].

\section{Genotyping techniques}

Detecting genetic variants of the parasite is important for studying the molecular epidemiology of the disease, and can make it possible to identify the source of imported infections. In elimination settings, it is important to know where infections originate and whether they are indigenous or imported. If the infections are imported, genotyping techniques can help to identify the origin of importation. Because malaria parasites are genetically highly variable, genotyping has been particularly valuable in distinguishing between domestic and imported cases. Genotyping techniques are useful for not only determining the genetic diversity of the malaria parasite and better understanding the molecular epidemiology of the disease, but also for the early detection of emergence and resurgence in malaria regions in the control and elimination phase. It is also especially important to monitor the spread of parasite resistance by detecting resistant Plasmodium strain variants. Genetic diversity can be measured with a single drop of dried blood, and the assay is rapid and accurate [41]. Moreover, molecular epidemiology can not only identify the origin of infection, but can also provide information about the place and time of transmission, and thus shed light on the relationships between parasites in elimination settings [36]. Available research has previously used molecular parasite markers which are under selective therapeutic or immunological pressure. However, other $P$. falciparum and $P$. vivax multilocus markers that are not under any selection pressure are also available and can provide evidence of variability [15].

\section{Serological techniques}

Seroprevalence testing to detect antibodies, but not to diagnose acute infection, is a technique that has been used for a long time [36]. The technique has been standardized and improved, but still needs to be approved as a routine method for surveillance. Malaria antibody quantification is a method that can provide information about the exposure time to malaria in patients predisposed to contract the infection by measuring the antibody level. The introduction of recombinant antigen technology makes serology a rigorous, standardized method. Antibodies can be detected in blood samples obtained by finger prick, and large numbers of samples can be assayed simultaneously in a short time. However, although sample collection is easy, most malaria programs are not prepared to test samples; assistance from research centers maybe needed. The measurement of malaria antibody responses is fairly accurate and can provide some species information; this method is able to detect $P$. falciparum, $P$. vivax and other species of malaria in a single sample, though cross-reactivity can occur. For epidemiological purposes, seroprevalence data can provide information about malaria endemicity in a given area and can distinguish between regions with variable exposure if the parasites cannot be detected [44]. The examination of seroprevalence profiles for age-related groups may be used to track changes in disease transmission and to identify the risk of exposure associated with travel [36]. The lack of antibodies in residents can be used to document the success of elimination programs in some settings; however, antibodies can remain present for a few years after exposure to malaria [44]. In field settings, cooperation with research centers is needed for specimen analysis and also to interpret the results, particularly because standardized quality assurance measures are not widely available [16]. 


\section{Endorsing research priorities for malaria elimination}

Basic research with clinical and operational research

The recent focus on malaria eradication has inspired the research community through the Malaria Eradication Research Agenda (malERA), which has developed a significant consensus for reformulating the entire malaria research agenda [15]. The malERA has highlighted the importance of both basic science and operational research in the fight against malaria; it has recommended that the new emphasis on taking action and on operational and translational research should not reduce basic scientific research [45]. Scientists proposed that it is extremely unlikely that the endgame in the battle against malaria will be reached without an increased understanding of parasite biology and basic knowledge [45].

Both the Plasmodium parasite and the Anopheles vector are always evolving, and in addition, the human environment is constantly changing. Therefore, new research questions concerning basic, clinical, and operational topics will continually arise during the whole elimination process. Thus, active research studies of malaria, particularly those concerned with developing innovative tools, must continue until and after eradication is finally achieved. The policymakers in the malaria elimination programs should examine and learn lessons from the elimination initiatives for smallpox, poliomyelitis and tuberculosis, and consider the role research has played in these experiences. The nine research and development agendas according to malERA classify priority research areas in eight different themes. These include basic science and enabling technologies; drugs; vaccines; vector control; health systems and operational research; modeling; diagnoses and diagnostics; and monitoring, evaluation, and surveillance [15]. Key examples of critical research needs include the availability of in-vitro cultures of $P$. vivax, $P$. ovale, and $P$. malariae to aid studies of the biology of the liver stages; hypnozoites and sexual forms of these parasites; genotyping of parasite isolates; drugs for treatment and prophylaxis; vaccines targeting different stages of the parasite; analyses of transcription, proteome and metabolome; drug libraries; antigen identification; functional analysis of gene products; new vector control approaches; new approaches for assessing transmission at the community level; and a tool kit to determine the readiness of country health system to switch to elimination [15]. All these research objectives are feasible but not yet fully realized. Achieving these objectives would provide laboratory scientists and field workers with important new information.

Governmental and nongovernmental investment in malaria research

A strong assumption exists that when planning the elimination agenda for the next phase, governments have to do everything (i.e., employ all staff, provide all products and deliver all services); yet this is a demanding and unnecessary burden on governments [9]. Every eliminating or controlling country should involve competent nongovernmental partners whose help should be incorporated through effective collaborations [46]. Some have pointed to successful collaboration experiences in the mining industries, faith-based organizations and other groups which have been active in many malaria programs including research, and which could do much more [46]. The greatest concern expressed with regard to elimination is rebound or resurgence of the disease if consistency, funding and efficient interventions decline. According to Roll Back Malaria, "experience in malaria control shows that when the disease falls to low rates and is no longer an evident threat, political leadership, support, and investment diminish and interventions are often discontinued and consequently deadly epidemics might occur" [8]. Indeed, new approaches are required to sustain the necessary political commitment through community engagement and investment in research to eliminate malaria and prevent its re-emergence. As Feachem et al. 2010 have argued, the maintenance of an expensive public health intervention in the absence of disease is commonplace and thought to be a costeffective investment [9].

Developing research leadership in endemic countries

Rather than counting only on external researchers and foreign experts in malaria, the development of research leadership in endemic countries is not only a politically correct step, but an essential requirement for long-term success [45]. Some argue that this development will take time and require much more investment than at present. Although it might be tempting to use external quick fixes, such an approach would be fundamentally misguided [45]. For many years, malaria elimination campaigns and research plans and activities were dominated by the views of a few scientists and policy makers who lived far removed from the dilemma. The main challenges to elimination include insufficient technical capability to inform malaria elimination programs and activities at the national level, and shortages of financial and 
experienced human resources in the region. Endemic countries should acquire human resource capabilities along with other logistic, financial, and operational rapid response capacities to deal with emergencies. Special consideration should be given to personnel training in malaria elimination at the local and national levels. For an improved malaria eradication agenda to have a chance to succeed, it will be essential for endemic countries to train malaria scientists in the various disciplines needed in an elimination program. Substantial efforts to train and authorize local personnel, along with measures to sustain research competence and elimination capacity in endemic countries, are crucial for the success of renewed control efforts and for the eventual elimination of malaria.

\section{Prioritizing research topics in malaria elimination Diagnostics and treatment research}

In many places throughout the world, the elimination of $P$. vivax poses particular challenges to countries eliminating malaria $[9,14]$. Safety problems with primaqune in patients with G6PD deficiency as well as the short half-life of this drug create difficulties in making clear recommendations for use of the only available radical treatment agent. Alternatives radical drugs are crucial to conquer this key obstacle to $P$. vivax elimination $[35,36]$. Research at the current stage should focus on three technologies: improving novel rapid diagnostic tests with higher diagnostic accuracy for $P$. vivax $[9,43]$, improving point-of-care tests for G6PD deficiency which can accurately detect high-risk individuals in field environment, and more effective, efficient and safer treatment than primaquine for $P$. vivax. [35]. For $P$. falciparum, more research in different endemic regions is required to provide stronger evidence of the benefits of a single dose of primaquine as a transmission-blocking agent versus the risk of hemolytsis [31,36].

\section{Vector and epidemiological research}

The first priority is recognizing the importance of going beyond epidemiology and considering renewed support for vector research [45]. Although malaria is a vector-borne disease, there is insufficient emphasis on research that examines various aspects of vector biology [15]. Studying vector biology would make it possible to establish the most effective vector control measures, which might be necessary to completely halt transmission. Second, as malaria transmission declines, understanding the heterogeneity in malaria transmission becomes crucial, and innovative approaches are needed in the field and in modeling. An important issue is how to define, monitor, and respond to the risk of resurgence in areas where malaria control has been achieved but the risk of reemergence remains high [45]. Additional research is required on mass drug administration and screening and its possible role and feasibility in real settings [45]. Among the aims of this research are identifying the best drugs, their dose and duration, and assessing safety concerns, affordability, access, effectiveness, and coverage [36].

Research on the impact of economic growth on malaria

Experts observed the effect on malaria of the average annual rate of increase in gross domestic product (GDP) per person in 32 countries that implemented malaria elimination from 1998 to 2008, and found a $3.3 \%$ increase. They also found that during this time, GDP decreased in only three countries [9]. This economic growth is believed have made many changes possible at the individual and countrywide levels. Changes in living conditions, work patterns, housing and overall conditions tended to affect a country's receptivity to malaria [9]. For example, a study in South Africa found that increased income and modern housing were significantly associated with a lower malaria risk [47].

To achieve malaria elimination in a region, the use and maintenance of monitoring, learning and evaluation (MLE) systems in malaria eradication programs requires a certain level of infrastructure. For example, an efficient communication network, one of the required infrastructures, is absent in some lowresourced countries. In some remote parts of Africa, landline phones, fast internet access, computers and paved roads are not readily available for the effective transfer of disease information [48]. Also, mobile phones and short message service (SMS) technology can form the basis of synchronized disease detection systems and integrated intervention efforts [49]. These communication tools can allow researchers to effectively collect and share data and enhance the routine reporting of malaria parameters such as antimalaria drug supplies at health facilities [49]. Nevertheless, more research is needed on the effect of economic growth on malaria elimination, on countrylevel health systems and circumstances and on their readiness to introduce and sustain novel programs and interventions [34]. 


\section{Vaccine research}

In areas where low-endemic control has been achieved but the potential for transmission remains high, an effective vaccine could play a new role by providing immunity to at-risk populations who no longer acquire natural immunity [50]. Malaria vaccine development has long been hindered by two main issues: the life cycle of the parasite is complicated, and the disease affects mainly people in low-income countries [51]. A further issue that has been highlighted is genetic polymorphism, especially in connection with a blood-stage malaria vaccine. WHO organized the Malaria Vaccines Advisory Committee (MALVAC) scientific forum in 2012 to review the global malaria vaccine portfolio; the committee suggested that "antigens with extensive genetic diversity should not transition into vaccine development pipelines unless constructs inducing cross-reactive immune responses have been produced" [52]. Children constitute the major at-risk group with higher fatality rates in these countries; embarrassingly, this group has no political traction in global health and therefore, the return on investment was seen as unlikely to satisfy sharing partners and pharmaceutical companies [51].

However, there are recent promising advances in this area, which may open a new chapter in the fight against malaria. The RTS,S plus adjuvant AS01 is a first generation pre-erythrocyte stage vaccine. This vaccine has been in development for about 20 years at a cost to sponsors and donors of almost $£ 500$ million [51]. Thus far it has shown modest short-term effectiveness [50]. In 2009, this vaccine entered a phase three clinical trial: 16,000 children in 11 African countries have participated, and scientists hope that this research will lead to the development of the first licensed malaria vaccine in 2013 [50].

Experts see that a serious investment in a broad research and development strategy aimed at producing an effective malaria vaccine is urgently needed. The next generation of pre-erythrocyte vaccines, a transmission-blocking vaccine, and a vaccine targeted against $P$. vivax are developmental steps that the world has urgently requested [51]. In this respect, the Bill and Melinda Gates Foundation has pledged US\$ 20 billion for the decade of vaccines. But greater commitment by the pharmaceutical industry and true public-private partnerships are needed to support the desperate call from countries to help solve one of their greatest health problems [50]. Hence, experts believe that the international health community must show more ambition and accountability in the struggle against malaria beyond its current control targets [51].

\section{Conclusion}

Controversies surrounding the benefits of the global malaria eradication goal show that experts are divided on this issue. The consensus seems to be that malaria elimination as a long-term goal is still important and eradication is worthy and achievable, but efforts must be conducted with balance, modesty, and rigorous examination [36]. However, elimination efforts should not be implemented at the expense of malaria control or the life-saving goal of decreasing morbidity and mortality rates of malaria in highly endemic settings [9]. Instead, it is important for elimination efforts to initially control malaria until the disease no longer constitutes a major public health problem, and this must be the central, most urgent target for sustained public and private investment worldwide.

The decision to eliminate malaria requires thoughtful consideration of all risks, benefits, and challenges [9]. The risks include the resurgence of malaria if elimination efforts wane, and the risk of emergence of new pathogens as a consequence of eradicating a microbe that has accompanied humanity for centuries. Challenges include improving the methods of diagnosis, treatment, vector control, surveillance and cross-country cooperation, and securing continuous commitments and resources to achieve and maintain elimination [36]. Research priorities include basic, clinical, and epidemiological studies aimed at increasing our understanding of the biology of the parasite and vector, along with the development of new generations of diagnostics, drugs, insecticides, and vaccines [45].

Finally, many believe that taking the path to malaria elimination will be hard work, and will require considerable time and huge amounts of money to achieve, but is nonetheless still possible and worthwhile [53-54]. Conversely, some believe that despite all these efforts, malaria will likely not be eliminated.

\section{Search strategy}

Data for this review were identified by searching PubMed up to April 2012 with the terms "elimination," "eradication," "control," "malaria," "plasmodium," and combinations of these, and by searching references from relevant articles. Other articles were identified through searches of the 
authors' own files, concerned-scientific conferences, WHO regional websites, the World Malaria Report, Roll Back Malaria, the Malaria Site on CDC, the Malaria elimination Group (MEG), and the All-party Parliamentary Group on Malaria. Malaria Collection articles in major journals such as The Lancet were searched, including collections of original research, expert comment and authoritative review. Articles in English were selected.

\section{Acknowledgements}

We thank K. Shashok (AuthorAID in the Eastern Mediterranean) for improving the English in the manuscript.

\section{References}

1. Henderson DA (2009) Smallpox: the death of a disease. The inside story of eradicating a worldwide killer. Amherst, New York: Prometheus Books 334 p.

2. Editorial (2009) Can malaria be eliminated? Lancet 374: 1794.

3. Molyneux DH, Hopkins DR, Zagaria N (2004) Disease eradication, elimination and control: The need for accurate and consistent usage. Trends Parasitol 20: 347-351.

4. WHO (2008) Global malaria control and elimination: Report of a technical review. Global Malaria Control and Elimination Meeting. Available: http://malaria.who.int/docs/elimination/MalariaControlElimin ationMeeting.pdf. Accessed 4 February 2012.

5. Centers for Disease Control and Prevention (2006) Locally acquired mosquito-transmitted malaria: A guide for investigations in the United States. MMWR Recomm Rep 55: $1-9$.

6. Cohen JM, Moonen B, Snow RW, Smith DL (2010) How absolute is zero? An evaluation of historical and current definitions of malaria elimination. Malar J 9: 213.

7. WHO (2007) Malaria elimination: a field manual for low and moderate endemic countries. Geneva: World Health Organization. Available: http://whqlibdoc.who.int/publications/2007/9789241596084_ eng.pdf. Accessed 4 April 2012.

8. Roll Back Malaria. Global Malaria Action Plan: For a malaria free world. Available: http://www.rollbackmalaria.org/gmap/gmap.pdf. Accessed 12 April 2012.

9. Feachem RGA, Phillips AA, Hwang J (2010) Shrinking the malaria map: progress and prospects. Lancet 367: 1566-1578.

10. Wernsdorfer WH, Hay SA, Shanks GD (2009) Learning from history. In Feachem RGA, Phillils AA, Targett GA, eds. Shrinking the malaria map: A prospectus on Malaria Elimination. San Francisc: the Global Health Group, Global Health Sciences, University of California, San Francisco. 95107.

Available: http://www.malariaeliminationgroup.org/sites/default/files/fil euploads/AProspectusonMalariaEliminationCh6.pdf. Accessed 25 March 2012.

11. Payne D (1987) Spread of chloroquine resistance in Plasmodium falciparum. Parasitol Today 3: 241-246.

12. Matthys B, Sherkanov T, Kariov SS (2008) History of malaria control in Tajikistan and rapid malaria appraisal in an agroecological setting. Malar J 7: 217.
13. Feachem RGA and The Malaria Elimination Group (2009) Shrinking the Malaria Map: A Guide on Malaria Elimination for Policy Makers. $1^{\text {st }}$ edition. San Francisco: The Global Health Group, Global Health Sciences, University of California, San Francisco 67 p.

14. Feachem R, Sabot O (2008) A new global malaria eradication strategy. Lancet 371: 1633-1635.

15. malERA.Malaria eradication research agenda. Available: http://malera.tropika.net/. Accessed 25 June 2012.

16. MEG. Malaria Elimination Group. Available: www.malariaeliminationgroup.org. Accesses 15 June 2012.

17. All-Party Parliamentary Group on Malaria and Neglected Tropical Diseases. The control of malaria 2005-15: progress and priorities towards eradication. Available: http://appmgmalaria.org.uk/upload/ 20Report\%202010.pdf. Accessed 15 June 2012.

18. The Global Fund to Fight AIDS, Tuberculosis and Malaria. The global fund 2010: innovation to impact. Available: http://www.theglobalfund.org/

documents/replenishment/2010/Global_Fund_2010_Innovatio n_and_Impact_en.pdf. Accessed 15 June $201 \overline{2}$.

19. Murray CJL, Rosenfeld LC, Lim SS (2012) Global malaria mortality between 1980 and 2010: a systematic analysis. Lancet 379: 413-431.

20. Sabot O, Cohen JM, Hsiang MS (2010) Costs and financial feasibility of malaria elimination. Lancet 376: 1604-1615.

21. Malaria Elimination Initiative. UCSF Global Health Group. Available:

http://globalhealthsciences.ucsf.edu/GHG/malaria_eliminatio n.aspx. Accessed 15 June 2012.

22. Teutsch SM, Churchill RE (2000) Prnciples and Practice of Public Health Surveillance. $2^{\text {nd }}$ edition. Oxford: Oxford University Press $248 \mathrm{p}$.

23. Zanzibar Malaria Control Program. Malaria elimination on Zanzibar: a feasibility assessment. Available: http://www.malariaeliminationgroup.org/malaria-eliminationzanzibar-feasibility-assessment. Accessed 15 June 2012.

24. Tatem AJ, Smith DL, Gething PW, Kabaria CW, Snow RW, Hay SI (2010) ranking of elimination feasibility between malaria-endemic countries. Lancet 376: 1579-1591.

25. WHO (2010) Guidelines for the treatment of malaria. Geneva: World Health organization. Available: http://www.who.int/malaria/publications/atoz/ 9789241547925/en/index.html. Accessed 16 June 2012.

26. Elmes NJ, Nasveld PE, Kichener SJ, Kocisko DA, Edstein MD (2008) The efficacy and tolerability of three different regimens of tafenoquine versus primaquine for post-exposure prophylaxis of Plasmodium vivax malaria in the Southwest Pacific. Trans R Soc Trop Med Hyg 102: 1095-1101.

27. Vale N, Moreira R, Gomes p (2009) Primaquine revisited six decades after its discovery. Eur J Med Chem 44: 937-953.

28. Beutler E, Duparc S, the G6PD Deficiency Working Group (2007). Glucose-6-phosphate dehydrogenase deficiency and antimalarial drug development. Am J Trop Med Hyg 77: 779789.

29. Shekalaghe SA, ter Braak R, Daou M (2010) Haemolysis after a single dose of primaquine co-administered with an artemisinin is not restricted to glucose-6-phosphate dehydrogenase (G6PD A-variant) deficient individuals in Tanzania. Antimicrob Agents Chemother 54: 1762-1768.

30. International Artemisinin Study Group (2004) Aretsunate combinations for treatment of malaria: meta-analysis. Lancet 363: 9-17. 
31. White NJ (2008) The role of anti-malarial drugs in eliminating malaria. Malar J 7 Suppl 1: 8.

32. Pukrittayakamee $\mathrm{S}$, Imwong $\mathrm{M}$, Singhasivanon $\mathrm{P}$, Stepniewska K, Day NJ, White NJ (2008) Effects of different antimalarial drugs on gametocyte carriage in $P$. vivax malaria. Am J Trop Med Hyg 79: 378-384.

33. Galappaththy GNL, Omari AAA, Tharyan P (2007) Primaquine for preventing relapses in people with Plasmodium vivax malaria. Cochrane Database Syst Rev 7. CD004389.

34. WHO (2009) World malaria report. Available: http://whqlibdoc.who.int/publications/2009/9789241563901_ eng.pdf. Accessed 16 June 2012.

35. 35. WHO/WPRO (2004) International Workshop on the Control of Vivax Malaria in East Asia. World Health Organization, Regional Office for the Western Pacific. Available:

http://www.wpro.who.int/internet/resources.ashx/MVP/ Vivax Mal East+Asia.pdf. Accessed 16 June 2012.

36. Moonen B, Cohen JM, Snow RW (2010) Operational strategies to achieve and maintain malaria elimination. Lancet 376: 1592-1603.

37. Kelly-Hope L, Ranson H, Hemingway J (2008) Lessons from the past: managing insecticide resistance in malaria control and eradication programmes. Lancet Infect Dis 8: 387-389.

38. WHO/EMRO (2007) Guidelines on prevention of the reintroduction of malaria. Geneva: World Health Organization Regional Office for the Eastern Mediterranean. Available: $\quad$ http://www.emro.who.int/dsaf/dsa742.pdf. Accessed 25 June 2012.

39. WHO (1956) The World Health Organization and malaria eradication. Geneva: World Health Organization. Available: http://whqlibdoc.who.int/publications/2008/9789241596756 eng.pdf. Accessed 25 June 2012.

40. Govella NJ, Okumu FO, Killeen GF (201) Insecticide-treated nets can reduce malaria transmission by mosquitoes which feed outdoors. Am J Trop Med Hyg 82: 415-419.

41. Ndao M, Bandyayera E, Kokoskin E, Gyorkos TW, MacLean JD, Ward BJ (2004) Comparison of blood smear, antigen detection, and nested-PCR methods for screening refugees from regions where malaria is endemic after a malaria outbreak in Quebec, Canada. J Clin Microbiol 42: 2694-2700.

42. Taylor SM, Juliano JJ, Trottman PA (2010) High-throughput pooling and real-time PCR-based strategy for malaria detection. J Clin Microbiol 48: 512-519
43. Wilson ML (2012) Malaria rapid diagnostic tests. Clin Infect Dis 54: 1637-1641.

44. Corran P, Coleman P, Riley E, Drakeley C (2007) Serology: A robust indicator of malaria transmission intensity? Trends Parasitol 23: 575-582.

45. Marsh K (2010) Research priorities for malaria elimination. Lancet 376: 1626-1627.

46. WHO (2000) The World Health Report — health systems: Improving performance. Available: http://www.who.int/whr/2000/en/whr00_en.pdf. Accessed 16 June 2012.

47. Coleman M, Mabaso ML, Mabuza AM, Kok G, Coetzee M, Durrheim DN (2009) Household and microeconomic factors associated with malaria in Mpumalanga, South Africa. Trans R Soc Trop Med Hyg 104: 143-147.

48. Jerome A (2011) Infrastructure, economic growth and poverty reduction in Africa. J Infrastructure Development 3: 127-151.

49. Asiimwe C, Gelvin D, Ben Amor Y, Quinto E, Katureebe C, Sundaram L, Bell D, Berg M (2011) Use of an innovative, affordable, and open-source short message service-based tool to monitor malaria in remote areas of Uganda. Am J Trop Med Hyg 85: 26-33.

50. Moorthy VS (2010) The elusive malaria vaccine: Miracle or mirage? Lancet ID 10: 81-82.

51. Editorial. Malaria (2010) More ambition and accountability please. Lancet 375: 1407.

52. Vannice KS, Brown GV, Akanmori BD, Moorthy VS (2012) MALVAC 2012 scientific forum: accelerating development of second-generation malaria vaccines. Malar J 9: 372.

53. Rodriguez-Morales AJ (2008) Malaria: an eradicable threat? J Infect Dev Ctries 2: 1-2.

54. Das P, Horton R (1981) Malaria elimination: Worthy, challenging, and just possible. Lancet 376: 1515-1517.

\section{Corresponding author}

Amal A. El-Moamly

Department of Medical Parasitology

Faculty of Medicine, Suez Canal University

Round Road, Ismailia, Postal Code 41522, Egypt

Phone: 00201115338807

E-mail: drama18@hotmail.com

Conflict of interests: No conflict of interests is declared. 